\title{
CLINICAL STUDY OF ANTERIOR SEGMENT FINDINGS IN PSEUDOEXFOLIATION SYNDROME
}

\author{
Krishnadeo Nalawade1, Smita Javadekar ${ }^{2}$
}

${ }^{1}$ Assistant Professor, Department of Ophthalmology, Prakash Institute of Medical Research \& Sciences, Prakash Nagar, Maharashtra. 2Professor \& HOD, Department of Ophthalmology, Prakash Institute of Medical Research \& Sciences, Prakash Nagar, Maharashtra.

ABSTRACT
BACKGROUND
Pseudoexfoliation syndrome (PEX) is a clinical entity characterised by the deposition of whitish-grey extracellular fibrins in the
anterior chamber of the eye. It may complicate the cataract surgery.
The aim of the study is to find out various anterior segment findings in patients having senile cataract with Pseudoexfoliation
syndrome.

\section{MATERIALS AND METHODS}

A cross-sectional study was conducted from December 2015 to December 2016 by Ophthalmology Department, Prakash Hospital \& Research Centre, Islampur. Patients with senile cataract attending Outpatient Department of Ophthalmology were included in the study. Complete clinical evaluation, with detailed slit-lamp biomicroscopy under maximal mydriasis with tropicamide $1 \%$ and Phenylephrine combination was performed. Undilated Gonioscopy was done in all cases. Diagnosis of PEX was based on the presence of grey flakes of PEX material on the anterior lens capsule or at the pupillary margin or both. Statistical analysis of the data was done by using Chi square test ( $\chi^{2}$ test). The level of significance was fixed at $p$ value less than 0.05 , i.e. $p<0.05$ was taken as statistically significant.

\section{RESULTS}

Total 300 patients were evaluated for cataract surgery out of which 165 were female \& 135 were male. $10 \%$ males (30) \& $15 \%$ (45) females were having PEX. PEX was significantly more common in older age group. Incidence of nuclear cataract (86.66\%) was significantly more in patients having PEX. PEX deposits on pupillary margin (80\%), poor mydriasis (69.33\%), \& PEX deposits on anterior lens capsule (94.6\%) were significantly observed $(\mathrm{P}<0.05 \%)$. IOP $>21 \mathrm{~mm}$ was present in $26(34.6 \%)$ cases. PEX deposits on trabecular meshwork was seen in 25 (33.33\%) cases. Phacodonesis was present in $11(14.6 \%)$ cases. Peripupillary atrophy was seen in $7(9.3 \%)$ cases. Pigment dispersion was seen in $8(6 \%)$ cases. Corneal endothelial deposits was seen in only one $(1.3 \%)$ case.

\section{CONCLUSION}

Pseudoexfoliation syndrome is common in advanced age group. PEX material is commonly seen on pupillary border, anterior lens capsule, zonules and trabecular meshwork. Nuclear and hard cataract is commonly seen in these patients. Due to deposition of PEX material, resistance in pupil for dilatation, open angle glaucoma, \& lens instability is common. All of these may complicate the cataract surgery and there is a need to take extra precautions in these patients prior to surgery.

\section{KEYWORDS}

Pseudoexfoliation, Anterior Segment, Pseudoexfoliation Material, Phacodonesis, Trabecular Meshwork, Glaucoma, Cataract, Zonular Weakness.

HOW TO CITE THIS ARTICLE: Nalawade K, Javadekar S. Clinical study of anterior segment findings in pseudoexfoliation syndrome. J. Evolution Med. Dent. Sci. 2017;6(37):3034-3037, DOI: 10.14260/Jemds/2017/654

\section{BACKGROUND}

Pseudoexfoliation syndrome (PEX), as defined by Lindberg in 1917 , is a clinical entity characterised by the deposition of whitish-grey extracellular fibrins in the anterior chamber of the eye and is commonly observed in older-aged patients.[1,2] There is amyloid like material on the anterior lens capsule, zonules, ciliary body, pupillary margin of the iris, corneal endothelium, anterior vitreous and trabecular meshwork.[3,4,5]

Financial or Other, Competing Interest: None.

Submission 25-03-2017, Peer Review 23-04-2017,

Acceptance 01-05-2017, Published 08-05-2017.

Corresponding Author:

Dr. Krishnadeo Nalawade,

'Aditya', Onkar colony,

Peth Sangli Road,

Tal. Walwa, Sangli-415409.

E-mail: krishnaeye7261@gmail.com

DOI: $10.14260 /$ jemds $/ 2017 / 654$
Prevalence rate of PEX in patients scheduled for cataract surgery shows extensive variations in Ethiopia (39.3\%),[6] Estonia (35.4\%),[7] Finland (30.8\%),[8] Greece (28\%),[9] Presence of EM has been shown not only in the eyes but also in the heart, lungs, liver, kidneys and meninges by using light/electron microscopes and immunohistochemical/ biochemical methods. ${ }^{[2,10,11]}$ Complications associated with secondary glaucoma, cataract and cataract surgery may develop in the eyes of PEX patients; the frequent occurrence of hypertension (HT), angina, myocardial infarction (MI), stroke, hearing loss and retinal vein thrombosis in PEX cases suggests the systemic feature of PEX.[2,10,11]

Factors that lead to the development of PEX have not been fully identified. A LOXL1 gene polymorphism has been detected in PEX cases.[1,2] PEX is a complex disease and the impact of environmental factors are also being considered in the pathology of PEX.[1,2] 
The definition and recognition of PEX (that affects eyes and other organ systems) incidence in the population is important for the prevention of potential complications.[12]

Its prevalence increases steadily with age and is rarely seen before the age of 50.[5] Patients with pseudoexfoliation syndrome have higher rates of complications during and after cataract surgery.[5]

\section{Aim of the Study}

Find out various anterior segment findings in patients having senile cataract with Pseudoexfoliation syndrome attending Outpatient Department of Prakash Hospital \& Research Centre, Islampur over the period of one year.

\section{MATERIALS AND METHODS}

A cross-sectional study was conducted from December 2015 to December 2016 by Ophthalmology Department, Prakash Hospital \& Research Centre, Islampur. Patients with senile cataract attending Outpatient Department of Ophthalmology were included in the study.

After informed consent, complete clinical evaluation was done including age, sex, visual acuity (VA) with Snellen chart, intra-ocular pressure (IOP) by Applanation tonometry, fundus examination.

Detailed slit-lamp biomicroscopy under maximal mydriasis with tropicamide $1 \%$ and Phenylephrine combination was performed to assess the type and grade of cataract. Pupillary dilatation of less than $6 \mathrm{~mm}$ after instillation of above drops was considered as poor mydriasis. Cataracts were classified morphologically as nuclear sclerosis (NS), cortical and posterior subcapsular (PSC).

PXE deposits on pupillary margin as well as iris atrophy if present were noted. Any associated finding such as corneal endothelial deposits, pigment dispersion were also noted.

Undilated Gonioscopy was done in all cases to detect PEX material on trabecular meshwork.

Diagnosis of PEX was based on the presence of grey flakes of PEX material on the anterior lens capsule or at the pupillary margin or both.

Statistical analysis of the data was done by using Chi square test $\left(\chi^{2}\right.$ test). The level of significance was fixed at $p$ value less than 0.05 i.e. $\mathrm{p}<0.05$ was taken as statistically significant.

\section{RESULTS}

\begin{tabular}{|c|c|c|c|}
\hline & Male & Female & Total \\
\hline PEX + & 30 & 45 & 75 \\
\hline PEX - & 105 & 120 & 225 \\
\hline Total & $\mathbf{1 3 5}$ & $\mathbf{1 6 5}$ & $\mathbf{3 0 0}$ \\
\hline \multicolumn{3}{|c|}{ Table 1. Sex Distribution } \\
\hline
\end{tabular}

Total 300 patients were evaluated for cataract surgery out of which 165 were females \& 135 were males. $10 \%$ males (30) \& $15 \%$ (45) females were having PEX. There was no significant difference of incidence as far as sex is considered ( $p>.05 \%)$.

\begin{tabular}{|c|c|c|c|}
\hline Age & PEX + & PEX - & Total \\
\hline $50-60$ & 2 & 35 & 37 \\
\hline $60-70$ & 13 & 120 & 133 \\
\hline $70-80$ & 45 & 60 & 105 \\
\hline 80 Above & 15 & 10 & 25 \\
\hline Total & $\mathbf{7 5}$ & $\mathbf{2 2 5}$ & $\mathbf{3 0 0}$ \\
\hline \multicolumn{3}{|c|}{ Table 2. Age Distribution } \\
\hline
\end{tabular}

As far as age is considered PEX was significantly more common in older age group ( $\mathrm{P}<.05 \%)$. Out of 75 cases, 60 patients were having age above 70 years $\& 73$ patients were having age above 60 years.

\begin{tabular}{|c|c|c|}
\hline Type of Cataract & PEX + & PEX - \\
\hline Nuclear & 65 & 110 \\
\hline Cortical & 30 & 60 \\
\hline Posterior Subcapsular & 40 & 80 \\
\hline \multicolumn{2}{|c|}{ Table 3. Type of Cataract } \\
\hline
\end{tabular}

Incidence of nuclear cataract (86.66\%) was significantly more in patients having PEX.

Nuclear, cortical and posterior subcapsular opacities were also present in both PEX positive \& negative patients.

\begin{tabular}{|c|c|c|}
\hline Clinical Finding & PEX + & \% \\
\hline Corneal endothelial deposits & 1 & 1.3 \\
\hline PEX Deposits on Pupillary Margin & 60 & 80 \\
\hline Poor Mydriasis & 52 & 69.33 \\
\hline Peripupillary Atrophy & 7 & 9.3 \\
\hline PEX Deposits on Trabecular Meshwork & 25 & 33.33 \\
\hline PEX Deposits on Anterior Lens Capsule & 71 & 94.6 \\
\hline Phacodonesis & 11 & 14.6 \\
\hline Pigment dispersion & 8 & 6 \\
\hline IOP>21 mm & 26 & 34.6 \\
\hline Table 4. Various Clinical Findings \\
\hline
\end{tabular}

- $\quad$ PEX deposits on pupillary margin (80\%), poor mydriasis (69.33\%), \& PEX deposits on anterior lens capsule (94.6\%) were significantly observed findings (P $<0.05 \%)$.

- $\quad \mathrm{IOP}>21 \mathrm{~mm}$ was present in $26(34.6 \%)$ cases.

- PEX deposits on trabecular meshwork was seen in 25 (33.33\%) cases.

- Phacodonesis was present in $11(14.6 \%)$ cases.

- $\quad$ Peripupillary atrophy was seen in $7(9.3 \%)$ cases.

- $\quad$ Pigment dispersion was seen in $8(6 \%)$ cases.

- Corneal endothelial deposit was seen in only one $(1.3 \%)$ case. 
Clinical Photographs

Pseudoexfoliation on Lens

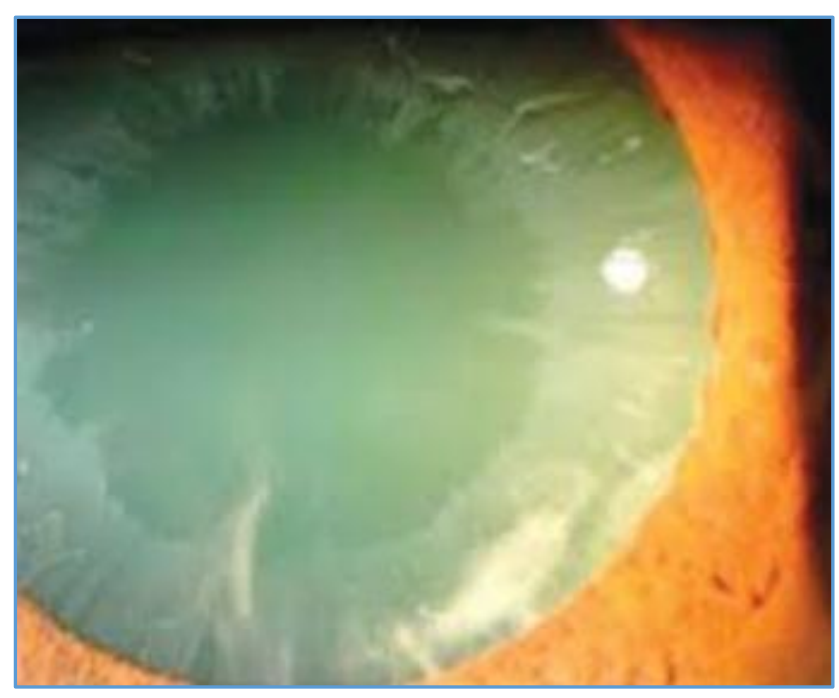

Pseudoexfoliation on pupil

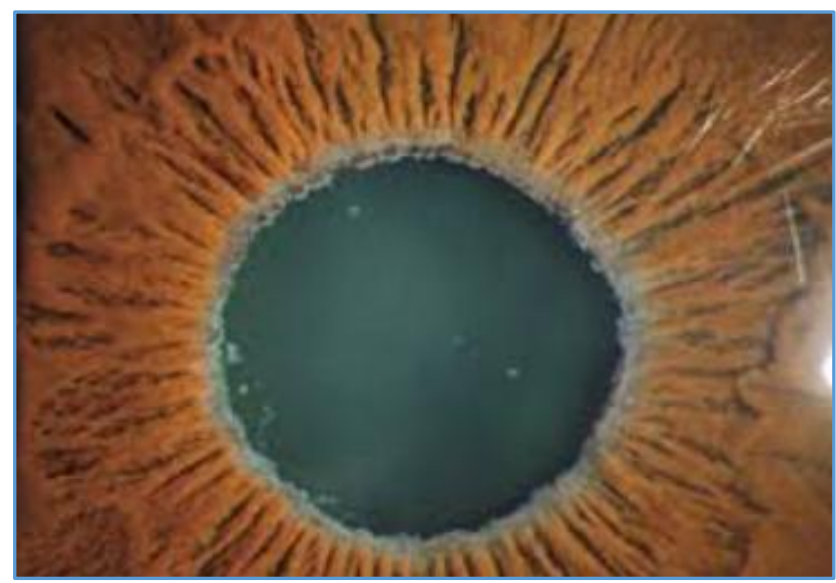

\section{DISCUSSION}

Pseudoexfoliation (PEX) syndrome is an age-related systemic disease with primarily ocular manifestations characterised by deposition of whitish-grey pseudoexfoliation fibrillogranular amyloid like material on the anterior lens capsule, zonules, ciliary body, pupillary margin of the iris, corneal endothelium, anterior vitreous and trabecular meshwork. ${ }^{[5,13]}$ Most common feature of PEX is whitish-grey flaky material on the pupillary border of the iris or on the anterior surface of the lens. ${ }^{[5,13]}$ In our study, we found PEX material on lens in $94.6 \%$, on pupillary border in $80 \%$ \& poor mydriasis in $69.33 \%$ cases.

The lens frequently demonstrates a "three-ring sign" on the anterior lens capsule which consists of a relatively homogenous central zone and a granular cloudy peripheral zone with a clear zone in between.[13]

Pigment loss from the iris sphincter region and its deposition on anterior chamber structures support the diagnosis in early cases. We found pigment dispersion in $6 \%$ cases, frequently PEX can lead to unexpected problems in management and during surgery so early diagnosis and meticulous slit-lamp examination is necessary.

Pseudoexfoliation is a risk factor for open-angle glaucoma. ${ }^{[5,14]}$ We found raised IOP more than $21 \mathrm{~mm}$ of HG in $34.6 \%$ cases. In these cases we will have to control IOP first $\&$ then we can proceed for combined surgery or Trabeculectomy first, then cataract surgery or if patient is compliant enough he can undergo cataract surgery and then take medical treatment for Glaucoma. But the best option is combined surgery in our rural area where patients are poor, they cannot come for followup frequently. It can also cause angle-closure glaucoma.

Rigid pupil not dilating more than 3 to $4 \mathrm{~mm}$ will require viscodilation or iris hooks can be kept ready for stretching the pupil. There were $69.33 \%$ cases with rigid pupil in our study.

Nuclear \& hard cataract is a common finding. We found $86.66 \%$ cases of nuclear cataract. While doing manual small incision cataract surgery care should be taken by taking adequate incision so that it may not damage the endothelium of the cornea. While doing Phaco surgery we may require extra power. Here also precaution should be taken not to damage the endothelium of the cornea.

Deposition of PEX material on zonules causes zonular weakness leading to subluxation or dislocation of lens. This manifests as phacodonesis.[13] We can see it on slit-lamp examination. There were $14.6 \%$ cases of phacodonesis in our study. In such cases we must keep capsular tension ring ready during surgery. There is also a risk of posterior capsular tear during surgery.[6]

Spontaneous subluxation of the lens can lead to angleclosure glaucoma due to pupillary and ciliary block.[5]

Exfoliation of fibrillogranular amyloid-like material has been found in many organs such as skin, heart, lungs, liver, kidney, gall bladder, blood vessels, extraocular muscle, connective tissue in the orbit, optic nerves and meninges suggesting that the pseudoexfoliation syndrome is not only an ocular disease but also a general disorder that involves the abnormal production of extracellular matrix material.[5] Recent investigations have shown the positive link between pseudoexfoliation and transient ischaemic attacks, stroke, heart disease and aneurysms of the abdominal aorta.[5] Although the exact aetiology of this condition as well as the exact structure of the material is still unknown, it is presumed that the production of pseudoexfoliation material is associated with abnormal metabolism of glycosaminoglycans and thus abnormalities of the basement membrane in the epithelial cells.

Pseudoexfoliation syndrome occurs in all areas of the world with varying frequency. Pseudoexfoliation syndrome is more common in females than in males ${ }^{[5]}$ and its prevalence increases steadily with age and is rarely seen before the age of 50. In our study, we could not found prevalence in females but it was observed that its incidence is significantly common in age group more than 50 years, so also its incidence increases as age advances. Jonasson et al reported a $10 \%$ annual increase for both open-angle glaucoma and pseudoexfoliation in persons of 50 years and over in Iceland.[14] The reported mean age ranges from 69-75 years.

Genetic factors influencing pseudoexfoliation has been explored considerably in the latest decade.[5] Recent genetic studies in multiple populations have identified the lysyl oxidase-like 1 (LOXL1) gene as a major contributor to the risk of developing pseudoexfoliation syndrome and pseudoexfoliation glaucoma. LOXL1 belongs to the lysyl oxidase family of extracellular enzymes that have multiple 
functions including the synthesis and maintenance of elastic fibres. However, the exact relationship between LOXL1 polymorphisms and the development of pseudoexfoliation has not been completely elucidated and thus the value of genetic testing for this disorder for the time being has not been validated.[11] In addition to heritable a number of nongenetic factors such as ultraviolet light, autoimmunity, slow virus infection, and trauma are supposed to have an implication in the development of pseudoexfoliation. It is possible that a combination of genetic and nongenetic factors may be involved.

\section{CONCLUSION}

Pseudoexfoliation syndrome is common in advanced age group.

PEX material is commonly seen on pupillary border, anterior lens capsule, zonules and trabecular meshwork.

Nuclear and hard cataract is commonly seen in these patients.

Due to deposition of PEX material on various tissues of anterior segment of eye resistant pupil for dilatation, open angle glaucoma, closed angle glaucoma and lens instability are common clinical implications. All of these may complicate the cataract surgery and there is a need to take extra precautions in these patients prior to surgery. Meticulous followup post surgery is also necessary for raised intraocular pressure and lens displacement. It is very important to do meticulous clinical examination prior to surgery in all patients having cataract so that PEX is not missed to avoid complications of cataract surgery.

\section{REFERENCES}

[1] Dewundara S, Pasquale LR. Exfoliation syndrome: a disease with an environmental component. Curr Opin Ophthalmol 2015;26(2):78-81.

[2] Anastasopoulos E, Founti P, Topouzis F. Update on pseudoexfoliation syndrome pathogenesis and associations with intraocular pressure, glaucoma and systemic diseases. Curr Opin Ophthalmol 2015;26(2):82-9.
[3] Naumann GO, Schlotzer-Schrehardt U, Kuchle M. Pseudoexfoliation syndrome for the comprehensive ophthalmologist. Intraocular and systemic manifestations. Ophthalmology 1998;105(6):951-68.

[4] Ritch R. Exfoliation syndrome. Chap 47. In: Ritch R, Shields MB, Krupin T. eds. The Glaucomas. $2^{\text {nd }}$ edn. Vol. 2. St Louis: Mosby 1996:993-1022.

[5] Kaštelan S, Tomic M, Kordic R, et al. Cataract surgery in eyes with Pseudoexfoliation (PEX) syndrome. J Clinic Experiment Ophthalmol 2013:S1-S5. http://dx.doi.org/10.4172/2155-9570.S1-009T

[6] Teshome T, Regassa K. Prevalence of pseudoexfoliation syndrome in Ethiopian patients scheduled for cataract surgery. Acta Ophthalmol Scand 2004;82(3 Pt 1):254-8.

[7] Kaljurand K, Puska P. Exfoliation syndrome in Estonian patients scheduled for cataract surgery. Acta Ophthalmol Scand 2004;82(3 Pt 1):259-63.

[8] Lumme P, Laatikainen L. Exfoliation syndrome and cataract extraction. Am J Ophthalmol 1993;116(1):515.

[9] Konstas AGP, Dimitrakoulias N, Kourtizidon 0, et al. Frequency of exfoliation syndrome in Greek cataract patients. Acta Ophthalmol Scand 1996;74(5):478-82.

[10] Holló G. Exfoliation syndrome and systemic cardiovascular diseases. J Glaucoma 2014;23(8 Suppl 1):S9-11.

[11] Samarai V, Samarei R, Haghighi N, et al. Sensoryneural hearing loss in pseudoexfoliation syndrome. Int J Ophthalmol 2012;5(3):393-96.

[12] Yildirim N, Yasar E, Gursoy H, et al. Prevalence of pseudoexfoliation syndrome and its association with ocular and systemic diseases in Eskisehir, Turkey. Int J Ophthalmol 2017;10(1):128-34.

[13] Majka CP, Challa P. Diagnosis and Management of Pseudoexfoliation Glaucoma. AAO, Eyenet Magazine 2006.

[14] Jonasson F, Damji KF, Arnarsson A, et al. Prevalence of open-angle glaucoma in Iceland: Reykjavik eye study. Eye (Lond) 2003;17(6):747-53. 